\title{
Human cystic echinococcosis in South Africa
}

\author{
Authors: \\ Benjamin Mogoye \\ Colin N. Menezes ${ }^{2}$ \\ Martin P. Grobusch ${ }^{2,3,4}$ \\ Kerstin Wahlers ${ }^{5}$ \\ John Frean ${ }^{1}$ \\ Affiliations: \\ ${ }^{1}$ National Institute for \\ Communicable Diseases, \\ National Health Laboratory \\ Service, South Africa \\ ${ }^{2}$ Department of Internal \\ Medicine, Chris Hani \\ Baragwanath Hospital \\ and University of the \\ Witwatersrand, South Africa \\ ${ }^{3}$ Institute of Tropical \\ Medicine, University of \\ Tübingen, Germany \\ ${ }^{4}$ Department of Internal \\ Medicine, University \\ Amsterdam, The Netherlands \\ ${ }^{5}$ Comprehensive Infectious \\ Diseases Center, University \\ Hospitals, Germany

\section{Correspondence to:} \\ Martin Grobusch \\ Email: \\ musoket@arc.agric.za \\ Postal address: \\ Department of Internal \\ Medicine, Chris Hani \\ Baragwanath Hospital \\ and the University of the \\ Witwatersrand, Faculty of \\ Health Sciences, 7 York Road, \\ Johannesburg 2193 \\ South Africa \\ How to cite this abstract: \\ Mogoye, B., Menezes, C.N., \\ Grobusch, M.P., Wahlers, K. \\ \& Frean, J., 2012, 'Human \\ cystic chinococcosis in \\ South Africa', Onderstepoort \\ Journal of Veterinary \\ Research 79(2), Art. \#469, \\ 1 page. http://dx.doi. \\ org/10.4102/ojvr.v79i2.469 \\ Note: \\ Proceedings of the \\ Conference of the Southern \\ African Centre for Infectious \\ Disease Surveillance 'One \\ Health' held at the National \\ Institute for Communicable \\ Diseases, Johannesburg, July \\ 2011.
}

Cystic echinococcosis (CE) is caused by the tapeworm, Echinococcus granulosus. The tapeworms resides in the small intestines of canids and the lifecycle involves both intermediate and definitive hosts. Humans are accidental intermediate hosts. Cystic echinococcosis is an economically important infection constituting a threat to public health, and is considered an emerging disease around the world. There are at least 10 Echinococcus strain types (G1-G10), each exhibiting diversity of morphology, development and host range. The epidemiology of CE is poorly understood in South Africa. A retrospective data analysis of the National Health Laboratory Service (NHLS) laboratory information system on echinococcosis serology, microscopy and histopathology results in eight provinces (excluding KwaZula-Natal) showed an overall positivity rate in submitted diagnostic samples of $17.0 \%(1056 / 6211)$, with the Eastern Cape (30.4\%), North West $(19.0 \%)$ and Northern Cape (18.0\%) provinces showing highest rates. The data showed considerable variability between provinces. The review also showed that most proven cases were negative on serology, implying that the actual number of patients could be underestimated. To our knowledge, no data exist about the prevalent strains of E. granulosus and this prospective study will attempt to fill that gap. The aim is to genotype strains causing the disease in South Africa. Two different polymerase chain reaction (PCR) methods will be used to respectively target the $12 \mathrm{~S}$ rRNA and nad 1 genes. To date, three samples have been genotyped as G1, G5 and G6; suggesting diversity of strains prevalent in the country, but more data is needed for a clearer picture. 